\title{
Pagsasalin at ebalwasyon ng mga panitikang Southern Leyteňo sa wikang Filipino
}

\author{
Mabalhin, Joel $\$ \\ Visayas State University, Philippines (joelqmabalhinbee7@gmail.com)

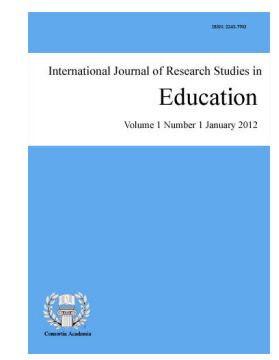

Accepted: 30 May 2021

ISSN: 2243-7703 Online ISSN: 2243-7711

OPEN ACCESS

\section{Abstract}

This research aims to translate and evaluate Southern Leyteño's Literature to Filipino Language. Descriptive analysis was used to get the frequencies, percentage and mean. A short story, drama, folktale and folk song which was originally written in Cebuano were selected. These were undergone in three translation stages: preparation, actual translation and evaluation of translated materials. The translation itself also goes through three phases: comparative-analysis, comprehension-check, and re-translation. In comparative analysis, six experts evaluated the material to know if there was a need for revision from the original text to the translated items. 40 secondary students, 40 college students and 12 Filipino teachers from Saint Joseph College did the comprehension check, and the final translation was done for the refinement of the materials. All student respondents in their analysis were all highly agreed, which means, translations of Southern Leyteño's literature was good. It was also discovered that gender and interest in literature has significant relationship due to disagreement of boys and girls when it comes to choice of reading materials. It is recommended further to use the translated articles in literature class. Literatures from other regions in the country should also be translated to Filipino language.

Keywords: Southern Leyteno's literature; Cebuano; folk tale; drama; folk song; short story 


\section{Pagsasalin at ebalwasyon ng mga panitikang Southern Leyteňo sa wikang Filipino}

\section{Introduksyon}

Inilalarawan ng panitikan ang ating lipunan at panlahing pagkakakilanlan. Ang ating kaugalian ay mababakas sa ating mga kuwentong bayan, alamat, epiko, awiting-bayan, kasabihan, bugtong, palaisipan at sinaunang-dula. Ang ating mga ninuno ay mayaman sa katitikan na nagbibigay ng kaisipan at nagtatampok sa kalinangan at kultura ng ating lahi. Tulad ng mga Southern Leyteňo na matatagpuan sa rehiyon, ay may mayaman din sa makabuluhang kasaysayan na pinag-uugatan ng iba't ibang panitikan na hanggang sa kasalukuyan ay sariwang-sariwa pa rin sa kanilang mga isipan at alaala ang lahat ng mga ito.

Isa sa mga namumukod-tanging tampok ng $\mathrm{K}$ to 12 Curriculum ang pagtuturo ng mga lokal na kayamanan ng isang rehiyon o tinatawag na lokalisasyon batay sa Department of Education (DepEd) (2015). Nakapaloob dito ang paggamit ng mga lokal na panitikan, kasaysayan, magagandang tanawin, pagkain, at mga pagdiriwang. Iniutos ng DepEd sa bawat rehiyon na bumuo na pangkat ng mga guro upang makabuo ng tinatawag na Division Curriculum Localization Matrix (DCLM). Nakikitaan ayon kay Egcas et. al. (2018), na mas madaling matuto ang mga mag-aaral kapag kabisado ang mga konseptong o mga halimbawang ginamit sa pagtuturo.

Kaya nga sinadya ng mananaliksik na pagtuunang pansin ang pagsasalin ng mga panitikang Southern Leyteňo, dahil nais niyang maibahagi ang kayamanang pampanitikan ng liping ito sa iba't ibang panig ng bansa. Sa sobrang dami at may kanya-kanyang wika at wikain ang bawat pangkat ng mga tao sa Pilipinas, malayong maunawaan ang mga panitikan na hindi nakasulat sa ating pambansang wika - ang Filipino. Ang pagsasalin na ito ay nakatuon sa isang maikling kuwento at dula ni Vestal (1993), awiting- bayan at kuwentong-bayan mula kay Lentejas (1980). Ayon kay Santiago (2003), ang pagsasaling-wika ay paglilipat sa pinagsalinang wika ng pinakamalapit na diwa at estilong nasa wikang isinalin. Dagdag pa ni Newmark (1988), ang pagsasaling-wika ay pagbibigay kahulugan ng isang text sa ibang wika sa paraang ninanais ng may-akda.

\subsection{Suliranin}

Pangunahing layunin ng pag-aaral na ito na maisalin at makita ang kaugnayan ng mga salik ng tagasagot at sa kanilang pagtataya sa panitikan ng mga Southern Leyteňo, katulad ng maikling kuwento, dula, kuwentong-bayan at awiting-bayan. At alamin ang mga salita sa mga panitikan na naisalin sa wikang Filipino, paano iniebalweyt ng mga mag-aaral sa sekundarya, kolehiyo at mga guro ang pagsasalin ng Panitikang Southern Leyteňo sa wikang Filipino, kung may makabuluhang pagkakaiba ang pagsusuri ng pagsasalin sa pagitan ng mga mag-aaral sa sekundarya at kolehiyo, at kung may makabuluhang kaugnayan ang mga salik sa tagasagot at ang pagsusuri ng mga panitikang Southern Leyteňo.

\section{Metodolohiya}

\subsection{Disenyo ng pag-aaral}

Ginamit sa pag-aaral na ito ang deskriptibo o palarawang pananaliksik. Ginawang batayan sa pagsasalin ng mga kuwento, dula, kuwentong-bayan at awiting bayan ang mga mungkahi ni Larson (2008). Binasa nang paulit-ulit ang mga napiling panitikan. Habang binabasa, inalam kung anong kaisipan ang nais iparating sa kabuoan, gayon din ang mga idyoma/tayutay at mga kultural na salita at ito'y itinala. Ginamit din ang modelo at teknik ni Newmark sa pagsusuri na lubos na nakatutulong sa mananaliksik sa pagsasalin ng kuwento tulad ng pagdaragdag, pagpapaikli, naturalisasyon, kultural na katumbas, pangsyunal na katumbas, adapsiyon. Modulasyon at kuplets.

94 Consortia Academia Publishing (A partner of Network of Professional Researchers and Educators) 
Gumawa ng unang pagsasalin pagkatapos mabasa nang paulit-ulit ang buong maikling kuwento, dula, kuwentong-bayan, at awiting-bayan. Ginawang tala-talata ang pagsasalin. Binasang muli at tiniyak ang kawastuhan ng salin kung kumpleto ang diwa, natural na daloy at himig ng pagpapahayag, pagkatapos inihahambing ang unang burador. Ibinigay sa dalawang tagasuri ang salin at ang orihinal na akda upang suriin ang paraan. Pagkatapos na masuri ng mga kritiko ang salin, nakapagbigay sila ng mga puna at mungkahi sa mga nakitang kaunting kalabuan para sa lalong ikaganda ng salin.

Upang makasiguro sa balidasyon ng ginamit na instrumento, ipinakita at ipinawasto ng mananaliksik sa mga subject specialist ang talatanungan upang ihingi ng mungkahi para sa anumang kakailanganing pagbabago at pag-aayos ng mga katanungan.

\subsection{Mga tagasagot}

Ang mga tagasagot sa pag-aaral na ito ay labindalawang (12) guro sa Filipino, apatnapung (40) mag-aaral sa junior hayskul, at apatnapung mag-aaral sa kolehiyo sa Saint Joseph College, Maasin City. Ang mga binanggit na mga kalahok ay sapat na upang ma-ebalweyt at masagot ang talatanungan, at maging makatotohanan at kapani-paniwala ang pananaliksik na ito at para na rin sa madaling pagkuha ng mga datos.

\subsection{Instrumentasyon}

Ang mananaliksik ay gumamit ng dalawang hakbang. Ang una ay pagsasalin ng mga nakalap na dula, maikling kuwentog-kwentong bayan at awiting- bayan ng mga Southern Leyteňo, at ipinasuri sa mga eksperto. Ang ikalawa ay ang pagsusuri ng salin, ang hakbang na ito ay isinagawa sa dalawang paraan: a) hambingang-suri (comparison check), at b). subok-pag-uunawa (comprehension check) batay kay De Guzman (2004). Sa ikalawang hakbang ng pag-aaral, ang mga tagasagot ay hiniling na sagutin ang mga talatanungan o bigyan ng rate ayon sa kanilang pananaw gamit ang eskalang lubos na sumang-ayon, sumasang-ayon, walang katiyakan, di-sumasang-ayon, at lubos na di-sumasang-ayon.

\subsection{Pangangalap ng datos}

Upang mas higit na maging maayos at mabilis ang pag-aaral na isinagawa, humingi ang mananaliksik ng pahintulot sa punong-guro sa hayskul at dekana sa kolehiyo ng Saint Joseph College na kinabibilangan ng mga guro at mag-aaral bago ipamahagi ang mga talatanungan at pasagutan sa mga kasangkot sa pag-aaral na ito.

\subsection{Pagsusuring istatistikal}

Upang masagot ang suliranin sa pag-aaral na ito, ang mga nakalap na datos ay kailangang gamitan ng mga sumusunod na instrumentong istatistikal:

Ang T-test ang siyang ginamit upang makita ang magkaibang pananaw ng mga tagasagot sa pagsasaling-wika na ginawa ng mananaliksik sa pagsusuri nito at makita ang kaugnayan sa pagitan ng mga salik ng tagasagot at sa pagsusuri ng mga isinaling akdang pampanitikan.

Ang limang puntos na iskala ay gagamitin upang mabigyang kahulugan ang mga datos: 5 - lubos na sumasang-ayon, 4-sumasang-ayon, 3-walang katiyakan, 2-di sumasang-ayon, at 1-lubos na di-sumasang-ayon.

\section{Resulta at talakayan}

Kabilang sa mga profayl ng mga mag-aaral at guro ang kanilang gulang, kasarian, antas o katungkulan (designation), kinawiwilihang asignatura at kinawiwilihang anyo ng panitikan. 
Mabalhin, J.

\section{Talahanayan 1}

Distribusyon ng mga tagasagot batay sa gulang, kasarian at antas/katungkulan

\begin{tabular}{cccc}
\hline & Sekundarya & Kolehiyo & Guro \\
\hline Gulang & $15-16100 \%$ & 23 pataas 58\% & 23 pataas $100 \%$ \\
& & $21-2212 \%$ & \\
Kasarian & Babae 72\% & $19-2023 \%$ & Babae 83\% $83 \%$ \\
Lalake 28\% & Lalake 17\% & Lalake 17\% \\
Antas & $100 \%$ & $100 \%$ & $100 \%$ \\
\hline
\end{tabular}

Ipinakikita sa talahanayan 1 na ang lahat ng tagasagot na mag-aaral sa sekundarya ay nabibilang sa gulang 15-16 na may kabuuang $100 \%$. Sa kolehiyo, ang may gulang $17-18$ ay 58\%, 23\% ay may gulang $19-20,12 \%$ na may gulang 21-22, at $7 \%$ na may gulang mula 23 pataas. At sa mga guro $100 \%$ na nabibilang sa gulang 23 pataas. Makikita din ang kasarian ng mga mag-aaral sa sekundarya, 28\% mga lalake, at $72 \%$ mga babae. Sa kolehiyo, $20 \%$ mga lalake, at $80 \%$ mga babae. At sa mga guro naman, $17 \%$ mga lalake, at $83 \%$ mga babae. Sa antas naman ng tagasagot, parehong $100 \%$ napabilang sa Grade 10 Junior HS, 2nd year sa Kolehiyo, at mga guro sa Filipino.

\section{Talahanayan 2}

Distribusyon ng mga tagasagot sa kinawiwilihang asignatura

\begin{tabular}{cccc}
\hline & Sekundarya & Kolehiyo & Guro \\
\hline Filipino & $15 \%$ & $38 \%$ & $100 \%$ \\
English & $25 \%$ & $30 \%$ & $0 \%$ \\
Math & $40 \%$ & $18 \%$ & $0 \%$ \\
Science & $10 \%$ & $5 \%$ & $0 \%$ \\
Aral. Pan & $5 \%$ & $5 \%$ & $0 \%$ \\
TLE & $5 \%$ & $2 \%$ & $0 \%$ \\
MAPEH & $0 \%$ & $2 \%$ & $0 \%$ \\
\hline
\end{tabular}

Malinaw na ipinakikita sa talahanayan 2 na sa mga tagasagot sa sekundarya, matematika ang may pinakamataas na umabot ng 40\%, ito'y nangangahulugang ang pagkawili sa matematika ay kakikitaan ng katalinuhan ng isang tao na siyang pinaniniwalaan ng mga mag-aaral sa sekundarya. Sa kolehiyo, $38 \%$ na ang kinawiwilihan ay Filipino. Ito ay nagsasaad na karamihan sa mga tagasagot ay nakapili na ng kanilang major at iyon ay ang Filipino na kanila namang gusto bilang mag-aaral na kumuha ng Bachelor of Secondary Education (BSEd- Filipino) Sa mga guro naman, 100 ay sa naturang asignatura ang kinawiwilihan, palibahasa'y mga guro sila sa Filipino na matagal ng nagtuturo ng asignaturang Filipino.

\section{Talahanayan 3}

Distribusyon ng mga tagasagot sa kinawiwilihang anyo ng panitikan

\begin{tabular}{cccc}
\hline & Sekundarya & Kolehiyo & Guro \\
\hline Maikling-Kuwento & $43 \%$ & $55 \%$ & $67 \%$ \\
Dula & $15 \%$ & $10 \%$ & $0 \%$ \\
Tula & $15 \%$ & $0 \%$ & $0 \%$ \\
Nobela & $5 \%$ & $15 \%$ & $17 \%$ \\
Kuwentong-bayan & $2 \%$ & $2 \%$ & $8 \%$ \\
Alamat & $18 \%$ & $13 \%$ & $8 \%$ \\
Pabula & $2 \%$ & $0 \%$ & $0 \%$ \\
Awiting-bayan & $0 \%$ & $5 \%$ & $0 \%$ \\
\hline
\end{tabular}

Malinaw na ipinakikita sa talahanayan 3, na $43 \%$ ang kinawiwilihang anyo ng mga mag-aaral sa sekundarya ay Maikling-kuwento. Ito ay nangangahulugang mas preperensya nila ang babasahing maaaring tapusing basahin sa isang upuan lamang at madaling maiugnay ang mga pangyayari sa kanilang totoong buhay. Sa mga mag-aaral sa kolehiyo ay 55\% na kinawiwilihan ay maikling-kuwento pa rin, nangangahulugang hindi pa rin nagbabago ang pananaaw ng mga mag-aaral maging sa ganitong antas. At sa mga guro, ang maikling-kuwento bilang 
Pagsasalin at ebalwasyon ng mga panitikang Southern Leyteňo sa wikang Filipino

kinawiwilihang babasahin ay 67\%. Ito'y nangangahulugang maging sa mga propesyunal ay mas higit pa ring kinawiwilihang babasahin na anyo ng panitikan ay maikling kuwento.

\section{Talahanayan 4}

Pagsusuri ng mga mag-aaral sa sekundarya tungkol sa pagsasalin ng panitikang Southern Leyteňo

\begin{tabular}{|c|c|c|c|}
\hline & Indikeytor sa talatanungan & Mean & SD \\
\hline 1 & Magkakatulad ang diwa ng orihinal at ng salin & 4.70 & 0.46 \\
\hline 2 & Ang salin ay kakikitaan ng sapat na pagkakaunawa sa wikang orihinal & 4.63 & 0.49 \\
\hline 3 & Malinaw ang pagpapahayag ng tunay na diwa na nais ipahatid sa sumulat & 4.63 & 0.49 \\
\hline 4 & Natumbasan nangtama ang orihinal na salita sa isinagawang pagsasalin & 4.38 & 0.63 \\
\hline 5 & Tuloy-tuloy ang daloy ng kaisipan sa pagbasa ng salin & 4.65 & 0.48 \\
\hline 6 & $\begin{array}{l}\text { Hindi nalalayo sa orihinal ang salin gaya ng idyomatikong salin, matatalinghagang salita, } \\
\text { mga salitang patambis, mga tayutay. }\end{array}$ & 4.25 & 0.59 \\
\hline 7 & $\begin{array}{l}\text { Angkop ang mga isinaling anyo ng panitikan para sa pinag-uukulang antas ng mga } \\
\text { mambabasa. }\end{array}$ & 4.65 & 0.48 \\
\hline 8 & Ang salin ay malinaw, natural at angkop sa target na mambabasa. & 4.73 & 0.45 \\
\hline 9 & Nananatili ang himig panahon ng awtor sa panahon ng tagasalin. & 4.78 & 0.48 \\
\hline \multirow[t]{2}{*}{10} & Nananatili ang istilo ng awtor sa salin & 4.70 & 0.61 \\
\hline & Kabuoan & 4.61 & 0.52 \\
\hline
\end{tabular}

Legend: 4.21 - 5.00 Lubos na sumasang-ayon, 3.41 - 4.20 Sumasang-ayon, 2.61 - 3.40 Walang Katiyakan, 1.81 - 2.60 Di-sumasang-ayon, 1.00 - 1.80 Lubos na di-sumasang-ayon.

Sampung (10) indikeytors ang ginamit upang malaman ang pagsusuri ng mga mag-aaral sa sekundarya. Ang data ay nagpapakita na ang mga tagasagot ay lubos na sumasang-ayon sa mga indikeytor na pinatutunayan ng kabuoang mean na 4.61. Ito ay nagsasaad na nauunawaang lubos ng mgatagasagot ang kanilang binasang salin, at hindi nalalayo ang diwa nito sa orihinal na mga akda.

\section{Talahanayan 5}

Pagsusuri ng mga mag-aaral sa kolehiyo tungkol sa pagsasalin ng panitikang Southern Leyteňo

\begin{tabular}{|c|c|c|c|}
\hline & Indikeytor sa talatanungan & Mean & SD \\
\hline 1 & Magkakatulad ang diwa ng orihinal at ng salin & 4.66 & 0.50 \\
\hline 2 & Ang salin ay kakikitaan ng sapat na pagkakaunawa sa wikang orihinal & 4.65 & 0.53 \\
\hline 3 & Malinaw ang pagpapahayag ng tunay na diwa na nais ipahatid sa sumulat & 4.60 & 0.50 \\
\hline 4 & Natumbasan nangtama ang orihinal na salita sa isinagawang pagsasalin & 4.30 & 0.52 \\
\hline 5 & Tuloy-tuloy ang daloy ng kaisipan sa pagbasa ng salin & 4.53 & 0.55 \\
\hline 6 & $\begin{array}{l}\text { Hindi nalalayo sa orihinal ang salin gaya ng idyomatikong salin, matatalinghagang salita, } \\
\text { mga salitang patambis, mga tayutay. }\end{array}$ & 4.38 & 0.54 \\
\hline 7 & $\begin{array}{l}\text { Angkop ang mga isinaling anyo ng panitikan para sa pinag-uukulang antas ng mga } \\
\text { mambabasa. }\end{array}$ & 4.53 & 0.55 \\
\hline 8 & Ang salin ay malinaw, natural at angkop sa target na mambabasa. & 4.63 & 0.54 \\
\hline 9 & Nananatili ang himig panahon ng awtor sa panahon ng tagasalin. & 4.53 & 0.51 \\
\hline \multirow[t]{2}{*}{10} & Nananatili ang istilo ng awtor sa salin & 4.58 & 0.55 \\
\hline & Kabuoan & 4.53 & 0.53 \\
\hline
\end{tabular}

Matapos makuha ang kabuoang mean na 4.53 lumalabas na ang mga mag-aaral sa kolehiyo ay lubos na sumasang-ayon sa mga inisa-isang indikeytor. Ito ay nangangahulugan na ang mga tagasagot ay nakakaintindi o nakauunawa nang lubos sa binasang salin at naiugnay ang diwa nito sa orihinal na akda.

\section{Talahanayan 6}

Pagsusuri ng mga guro sa pagsasalin ng panitikang Southern Leyteňo

\begin{tabular}{llcc}
\hline & Indikeytor sa talatanungan & Mean & SD \\
\hline 1 & Magkakatulad ang diwa ng orihinal at ng salin & 4.75 & 0.45 \\
2 & Ang salin ay kakikitaan ng sapat na pagkakaunawa sa wikang orihinal & 4.66 & 0.49 \\
3 & Malinaw ang pagpapahayag ng tunay na diwa na nais ipahatid sa sumulat & 4.58 & 0.51 \\
\hline
\end{tabular}


Mabalhin, J.

Talahanayan 6 ...pagtutuloy

\begin{tabular}{|c|c|c|c|}
\hline & Indikeytor sa talatanungan & Mean & SD \\
\hline 4 & Natumbasan nangtama ang orihinal na salita sa isinagawang pagsasalin & 4.33 & 0.49 \\
\hline 5 & Tuloy-tuloy ang daloy ng kaisipan sa pagbasa ng salin & 4.67 & 0.49 \\
\hline 6 & $\begin{array}{l}\text { Hindi nalalayo sa orihinal ang salin gaya ng idyomatikong salin, matatalinghagang salita, } \\
\text { mga salitang patambis, mga tayutay. }\end{array}$ & 4.42 & 0.51 \\
\hline 7 & $\begin{array}{l}\text { Angkop ang mga isinaling anyo ng panitikan para sa pinag-uukulang antas ng mga } \\
\text { mambabasa. }\end{array}$ & 4.67 & 0.49 \\
\hline 8 & Ang salin ay malinaw, natural at angkop sa target na mambabasa. & 4.75 & 0.45 \\
\hline 9 & Nananatili ang himig panahon ng awtor sa panahon ng tagasalin. & 4.58 & 0.51 \\
\hline \multirow[t]{2}{*}{10} & Nananatili ang istilo ng awtor sa salin & 4.58 & 0.51 \\
\hline & Kabuoan & 4.60 & 0.49 \\
\hline
\end{tabular}

Pinatutunayan ng nakuhang kabuoang mean na 4.60 na ang mga guro ay lubos na sumasang-ayon sa mga indikeytor tungkol sa pagsasalin ng panitikang Southern Leyteňo sa Wikang Filipino. Ito ay nangangahulugang ang ginawang pagsasalin ng mananaliksik ay lubos na katanggap-tanggap para sa kani-kanilang sariling pananaw sa pagsasagawa ng pagsasalin at sa paglalapat ng kanilang kaalaman hinggil sa mga simulain, pamamaraan at mga teorya sa pagsasaling-wika.

\section{Talahanayan 7}

Pagkakaiba ng pagsusuri sa sekondarya at kolehiyo tungkol sa pagsasalin ng mga panitikang Southern Leyteňo

\begin{tabular}{lccc}
\hline Pagsusuri ng dalawang pangkat & Comp t & Tabular t & Remarks \\
\hline & 0.26 & 1.684 & Di-makabuluhan \\
\hline
\end{tabular}

Ipinakikita sa itaas na matapos mapaghambing ang pagsusuri ng dalawang pangkat ang nakuhang $t$-value na 0.26 ay nagsasaad ng di makabuluhan ang pagkakaiba ng pagsusuri ng mga mag-aaral sa sekundarya at kolehiyo tungkol sa pagsasalin ng Panitikang Southern Leyteňo sa Wikang Filipino.

\section{Talahanayan 8}

Kaugnayan ng mga salik ng tagasagot sa sekundarya at ang pagsusuri ng mga panitikang Souther Leyteňo

\begin{tabular}{ccc}
\hline Mga salik & Pearson $-r$ & Remarks \\
\hline Gulang & .01 & Makabuluhang Ugnayan \\
Kasarian & .81 & Makabuluhang Ugnayan \\
Antas & .01 & Di-makabuluhang Ugnayan \\
Kinawiwilihang Asignatura & .99 & Makabuluhang Ugnayan \\
Kinawiwilihang anyo ng Panitikan & .37 & Makabuluhang Ugnayan \\
\hline
\end{tabular}

\section{Talahanayan 9}

Kaugnayan ng mga salik ng tagasagot sa kolehiyo at ang pagsusuri ng mga panitikang Southern Leyteňo

\begin{tabular}{ccc}
\hline Mga salik & Pearson $-r$ & Remarks \\
\hline Gulang & -.95 & Makabuluhang Ugnayan \\
Kasarian & -.58 & Makabuluhang Ugnayan \\
Antas & .01 & Di-makabuluhang Ugnayan \\
Kinawiwilihang Asignatura & .32 & Makabuluhang Ugnayan \\
Kinawiwilihang anyo ng Panitikan & .50 & Makabuluhang Ugnayan \\
\hline
\end{tabular}

*Ipinakikita ng Talahanayan 8 na ang mga salik ng mga mag-aaral sa sekundarya ay may kaugnayan sa kanilang pagsusuri gaya ng kasarian na may $r$ value $=.81$; kinawiwiwlihang asignatura nasa may $r$ value na $=.99$; ang kinawiwilihang anyo ng panitikan na may $r$ value na $=.37$. Subalit anng gulang at antas na parehong may $r$ value na $=.01$ ay walang kabuluhang kaugnayan sapagkat negligible lamang ang kanilang kaugnayan. Ayon sa paliwanag ni De Guzman (2004) sa kanyang pag-aaral na magkaiba ang lalake at babae sa kanilang kognitibong pananaw, kung may malaking epekto ito sa kanilang pag-iisip, at natuklasang mas magaling ang lalake sa babae sa paglutas ng suliranin na nangangailangan ng katalasan ng isip at wastong pangangatuwiran. 
**Sa mga mag-aaral sa kolehiyo, ang kanilang gulang, kasarian, kinawiwilihang asignatura at kinawiwilihang anyo ng panitikan ay may makabuluhang kaugnayan sa kanilang pagsusuri na pinatutunayan ng mga $r$-values na $=-.95=-.58=.32$ at $=.50$. Nangangahulugang ang mga tagasagot na mas mababa ang gulang ay maaaring sa kanilang pagtataya ay nababatay sa mga asignaturang tulad ng Mathematics, English at Science. Ito at nangangahulugang hindi pa masyadong napalalim ang pagpapahalaga sa sariling wika. Ayon kay Alibutod (2008) sa kanyang pag-aaral ang kognitibong pag-unlad ay nakukuha sa pamamagitan ng "maturational stages" kung saan ang proseso ng pag-iisip ay hulwaran ng pagbabago habang tumataas ang gulang.

\section{Talahanayan 10}

Pearson r na nagpapakita ng kaugnayan ng mga salik at pagsusuri ng mga panitikang Southern Leyteňo

\begin{tabular}{ccc}
\hline Mga salik & Pearson $-r$ & Remarks \\
\hline Gulang & .00 & Di-Makabuluhan \\
Kasarian & .50 & Makabuluhan \\
Antas/Katungkulan & .42 & Makabuluhan \\
Kinawiwilihang Asignatura & .00 & Di-Makabuluhan \\
Kinawiwilihang anyo ng Panitikan & .57 & Makabuluhan \\
\hline Legend: $* .00$ to \pm 20 Negligible/Di-Makabuluhan, \pm .21 to .40Di-masyadong makabuluhan, \pm .41 to \pm 70 Makabuluhang kaugnayan, \\
\pm .71 to \pm 1 Mataas ang kabuluhan.
\end{tabular}

Ipinakikita ng talahanayan 10 na ang mga salik ng mga tagasagot na guro ay may kaugnayan sa kanilang pagsusuri gaya ng kasarian na may $r=.50$; katungkulan na may $r=.42$; at kinawiwilihang anyo ng panitikan na may $r=.57$. Subalit ang gulang at kinawiwilihang asignatura na may parehong $r=.00$ ay walang kaugnayan sapagkat Di-makabuluhan ang pagkakaugnay nila.

\section{Konklusyon at rekomendasyon}

Sa pagsusuri sa pagitan ng mga mag-aaral sa sekundarya at kolehiyo, napatunayang lahat ay lubos na sumasang-ayon. Ito’y nangangahulugang malinaw ang pagkakasalin ng mga Panitikang Southern Leyteňo katulad ng maikling kuwento, dula kuwentong-bayan at awiting-bayan. Sa pagsusuri ng mga tagasagot, natuklasang ang kasarian ay may makabuluhang kaugnayan sa kadahilanang magkakaiba ang kinahihiligan ng mga babae at lalake pagdating sa babasahin, kung may pagkakapareho man ay maliit lamang ng bahagdan nito. Samakatuwid, ang mga haypotesis hinggil sa mga salik ay may makabuluhang kaugnayan sa pagsusuri ng mga Panitikang Southern Leyteňo gaya ng kasarian, kinawiwilihang asignatura at kinawiwilihang anyo ng panitikan.

Sa mga magsasagawa ng pagsasalin, maaaring suriing muli ang kasalukuyang pag-aaral at ang kahinaan at kalakasan ng ginawang pagsasalin, o gamitan ng ibang teorya, pagdulog o lapit, pamamaraan o teknik ng pagsasalin. Maaari ding gamitin ang salin bilang isa sa mga akdang pampanitikan sa lahat ng paaralan sa buong bansa upang maipaliwanag ang kultura ng mga Southern Leyteňo na nakapaloob sa kanilang panitikan. Ang ganitong pag-aaral ay lubusang inirerekomenda sa iba pang lugar lalo na sa mga mamamayang ang unang wika ay magkaiba sa wikang sinasalita sa Laguna at Maynila gaya ng Leyte, Cebu, Lanao Provinces, Iloilo, at iba pa.

\section{Sanggunian}

Alibutod, V. E. (2008). Multiple intelligences approach and academic performance of freshman students of hotel and restaurant management in general chemistry (Unpublished Thesis). Laguna State Polytechnic University, San Pablo City, Laguna.

De Guzman, N. C. (2004). Pagsasalin sa Filipino sa sinukman: Isang literaryong epikong kapampangan (Di-Nalathalang Disertasyon). Philippine Normal University, Manila.

DepEd Order 32, s. 2015. Retrieved from https://deped.gov.ph/2015/07/29/do-32-s-2015-adopting-the-indigenous-people-education-curriulum-fra mework/

Egcas, R. A., Tabotabo, M. T. L., \& Geroso, M. J. A. (2018). Localized curriculum on the reading achievement of Grade 8 students. Asia Pacific Journal of Multidisciplinary Research, 5, 137-138. 
Mabalhin, J.

Larson, M. L. (2008). Translation, theory and practice, tension and interdependence. John Benjamins Publishing.

Lentejas, A. B. (1980). Folklore as performance: A collection and study on thirty Cebuan version of Juan Pusong tale in Malitbog, Southern Leyte (Unpublished Thesis). University of San Carlos, Cebu City.

Newmark, P. (1988). A textbook of translation. Prentice-Hall

Santiago, A. O. (2003). Sining ng pagsasaling-wika. Rex Bookstore.

Vestal, R. B. (1993). Tigpamulak sa panahon. Anahawan. 